\begin{tabular}{l|l} 
REVISTA & $\begin{array}{l}\text { Revista Educación } \\
\text { ISSN: 0379-7082 } \\
\text { ISSN: 2215-2644 } \\
\text { revedu@ gmail.com } \\
\text { Universidad de Costa Rica } \\
\text { Costa Rica }\end{array}$
\end{tabular}

\title{
Preguntar-se en el oficio de educar: la importancia de la pregunta en formación docente
}

\author{
Aravena Domich, Marcela Angelina \\ Preguntar-se en el oficio de educar: la importancia de la pregunta en formación docente \\ Revista Educación, vol. 45, núm. 1, 2021 \\ Universidad de Costa Rica, Costa Rica \\ Disponible en: http://www.redalyc.org/articulo.oa?id=44064134033 \\ DOl: https://doi.org/10.15517/revedu.v45i1.41144
}

\section{(c) $(1) \Theta$}

Esta obra está bajo una Licencia Creative Commons Atribución-NoComercial-SinDerivar 3.0 Internacional. 


\section{Preguntar-se en el oficio de educar: la importancia de la pregunta en formación docente}

\section{The Education Profession and the Importance of Asking Questions while Teacher Training}

Marcela Angelina Aravena Domich

Universidad Metropolitana de Educación, Ciencia y

Tecnología - UMECIT, Panamá

domich2@yahoo.com

https://orcid.org/0000-0002-6951-0960
DOI: https://doi.org/10.15517/revedu.v45i1.41144

Redalyc: http://www.redalyc.org/articulo.oa?id=44064134033

Recepción: 23 Abril 2020

Aprobación: 06 Octubre 2020

\section{Resumen:}

Preguntar-se en el oficio de educar sitúa a la pregunta en un lugar privilegiado en la formación docente y en la relación de las temáticas que se presentan en la cotidianidad, tanto en la preparación como en el posterior ejercicio profesional. Preguntar-se cruza el artículo como idea fuerza, también lo hace en la formación docente, en los saberes, en la reflexión, en las relaciones de los y las docentes con la política educativa y la legalidad. Este ensayo revisa temáticas emergentes, sin la intención de agotarlos y los relaciona con interrogantes permanentes sobre la tecnología, las migraciones, los desplazados, la etnoeducación, los afrodescendientes, la educación para la paz, la sustentabilidad, la diversidad y género. El artículo finaliza considerando que poner a la pregunta como eje central de la formación y en la acción docente, permite plantearse desafíos en la labor y experiencia docente. Preguntar-se es poner a la población docente en formación en una tensión constante entre la teoría y la práctica, pero también en los cambios permanentes y, a veces, acelerados de la sociedad del siglo XXI.

Palabras Clave: Preguntas, Formación docente, Oficio de educar, Reflexión docente.

\section{ABstract:}

Self-questioning one's teaching performance should be a priority for teacher trainees, which aids in helping them to better assimilate the topics covered on a daily basis and prepare them for their professional lives. This article focuses on self-questioning as an inherent part of teacher training which strengthens knowledge, reflection and teacher relationships with education policies and observance of the law. Emerging topics are covered, although not exhausted, and questions are raised regarding technology, immigration, displaced individuals, ethno-education, afro-descendants, education for peace, sustainability and gender diversity. The conclusion emphasizes the need to ask oneself questions during training and on the job so that teachers may constantly challenge themselves and their work and teaching experience. Self-questioning forces teachers to break away from their comfort zone and continuously reflect on theory and practice as well as face the accelerated changes of 21 st century society.

KEYWORDS: Questions, Teacher Training, Education, Teacher Reflection.

\section{INTRODUCCIÓN}

la pregunta en el centro, desde la formación que permite transitar hasta ser docente, en los saberes, en la política y en la legalidad, en el rol docente, la tecnología, las migraciones, los desplazados, la etnoeducación, los afrodescendientes, la educación para la paz, la sustentabilidad, la diversidad y género, inquietudes necesarias para generar reflexión y ponerlas en acción. El artículo abordará tres partes esenciales, primeramente, los referentes respecto a la formación docente, los saberes y la reflexión en y para la acción. En un segundo momento, se abre a las preguntas sobre los temas emergentes ya señalados y que permiten la guía de todo el documento y, por último, las reflexiones finales. Algunas de las ideas fuerza que permiten cerrar este artículo dan cuenta de que el/la docente, tanto en su formación como luego de ella, no solo trabaja con temas pedagógicos, sino que debe hacer lectura de las cuestiones actuales de la sociedad; por tanto, lo ubica en un lugar privilegiado por su relación académica y social. La población docente en formación, no sólo debe 
preguntar-se sobre estas y otras temáticas, sino que debe acercarse, revisar, construir sus respuestas y hacerlas parte de la acción pedagógica. Son temas para el/la docente que no están aislados ni le son ajenos, sino que lo condicionan en una sociedad en permanente cambio.

\section{Formación DOCENTE}

La formación docente ha ocupado un lugar central en las sociedades, ha llegado a discutirse en varias cumbres y declaraciones mundiales. Sin duda, que ha sido, según Gil (2018), "un debate permanente, sobre si la formación del profesorado tiene efectos claros en los resultados de aprendizaje de los alumnos” (p. 258).

Ser un/a docente se forma desde el proceso inicial de estudio hasta la puesta en marcha de todos los saberes. Dependerá de distintas variables, entre ellas: la vocación, calidad en la formación y las capacidades individuales. El/la docente no sólo maneja contenidos, sino que además trabaja con metodologías y didácticas para lograr los objetivos de la propuesta curricular al interior del aula. Todo lo anterior, es parte de lo aprendido de manera articulada en los años de formación inicial. La importancia de la formación docente se refleja en la construcción de una identidad personal, lo que la convierte un proceso de gran complejidad.

Francesco Tonucci (2018) pone de manifiesto la necesidad de formación inicial docente, apoyando el rol docente como irremplazable y reseña esta importancia a través de sus propias teorías sobre niños y niñas, la educación y el entorno. En sus obras aborda la formación docente y a su población, en relación con las escuelas y en la preocupación de estos por desarrollar un espacio donde niños y niñas, estén a gusto.

El/la docente requiere de tiempo para aprehender saberes y capacidades. Para Sennett (2009) el oficio, en este caso para el/la docente, se realiza durante la formación y luego se desarrolla a través de la experiencia, donde el trabajo cotidiano y artesanal del crear día a día, de articular los conocimientos y abrir los caminos por donde transitar, hace del oficio docente una tarea inacabada y permanentemente incompleta. Como el artesano moldea su obra, la formación docente hará que con el tiempo se desarrolle el oficio de educar. La persona que domina un oficio y que se complace en hacer aquello que produce, en hacerlo bien, contribuyendo con este trabajo a dar un cierto sentido a la vida del que lo hace y una cierta felicidad. Los procesos de aprendizaje están basados en el hacer, en el pensar permanentemente sobre lo que se hace: cómo se hace, cómo puede hacerse mejor, para quién y para qué se hace. El oficio del/la docente se va elaborando en la formación temprana, se va construyendo con la práctica, lo que puede tardar años. El concepto de docente como artesano lo recoge también Larrosa (2020), quien señala que la relación que el/la docente tiene con los y las estudiantes, los textos, el aula y la pizarra, se relaciona con un trabajo manual, similar al que hace el artesano. En palabras de Fanfani (2006), la tarea docente sería un oficio sin obra, siempre en construcción, pero una obra material finalizada de un artesano que, en el caso del/la docente, sería un resultado inmaterial, complejo de medir, conocer y otorgarle un fin. Quizás eso es la complejización y debate permanente respecto al concepto de calidad de la enseñanza- aprendizaje.

¿Las y los docentes requieren saberes? Frente a esta pregunta, claramente se requiere formar y preparar al/la docente en los campos del saber, para posteriormente ejercer el desempeño docente. Es una tarea que cada país debe abordar y discutir sobre lo que se debe saber para ofrecerlo a la sociedad con la advertencia, que los conocimientos deben ser contextualizados y situados. Así, cobran importancia las preguntas sobre ¿cómo formar un/a buen/a docente?, ¿con qué conocimientos debe contar? ¿Cómo orientar al/la docente hacia una práctica con esos saberes en la sociedad actual?, ¿cómo orientar al/la docente hacia una práctica con esos saberes en la(s) sociedad(es) actual(es)?, ¿cómo incorporar en la formación docente y en las y los futuros docentes, la alta relevancia de la creación, poseer lúcidas lecturas de los nuevos sujetos contemporáneos (especialmente los niños, niñas y jóvenes) y la convicción en su actualización permanente? Estas interrogantes poseen una raíz común, todas refieren a los distintos saberes docentes.

Respecto de los saberes, señala Vezub (2016), son relevantes porque el conocimiento es primordial para la acción educativa que se constituye en la materia prima con la que las y los docentes deben trabajar. 
Así, la población estudiantil se preparan para recibir conocimientos relevantes que cada sociedad considera importantes. Cabe considerar que los conocimientos se fundan en el campo de la educación, la pedagogía, la didáctica, el currículum y la comunicación. El saber pedagógico, según Sánchez y González (2016):

Sería todo aquello que se puede decir, que se puede enunciar, acerca de la pedagogía o de las prácticas pedagógicas, que un sujeto - el maestro - pone en funcionamiento en el acto de enseñar, esto es, el fundamento de su práctica (p. 7).

Según los mismos autores, el saber estaría constituido por sujetos que enuncian discursos y prácticas relativas a la pedagogía, por disciplinas o campos disciplinares relacionados con la pedagogía, epistemologías, teorías sobre la educación y la política educativa, enfoques de aprendizaje, modelos pedagógicos, los modelos evaluativos, métodos de enseñanza, de relaciones e interacciones y que se ponen a disposición del/la docente para poder desarrollar la acción educativa.

Aportando a lo anterior, Tardif (2004) considera que el "saber docente es un saber plural, formado por una amalgama, más o menos coherente, de saberes procedentes de la formación profesional y disciplinarios, curriculares y experienciales" (p. 29). Además, el mismo autor, al examinar las relaciones de las y los docentes con estos saberes, realiza las siguientes preguntas que son interesantes en el sentido de proyectar la formación inicial docente:

¿Qué saben los docentes?; ¿qué saber es ese?; ¿'son meros 'transmisores' de saberes producidos por otros grupos?; ¿’producen uno o más saberes, en el ámbito de su profesión?; ¿ ¿cuál es su papel en la definición y en la selección de los saberes transmitidos por la institución escolar?; ¿cuál es su función en la producción de los saberes pedagógicos? (Tardif, 2004, p. 26)

Continúa Tardif (2004), señalando que los saberes y conocimientos, "antes de comenzar, oficialmente, a enseñar, los docentes ya saben, de muchas maneras, que es la enseñanza, por su historia escolar anterior” ( $p$. 15), considerando que el/la docente en formación se identifica con una pluralidad de saberes.

Siguiendo a Porta (2019), habría un conjunto de saberes en las y los docentes como los siguientes:

\begin{abstract}
Un repertorio o base de conocimientos particulares a la función del profesorado que lo diferencian de otros profesionales. Los profesores disponen de un amplio bagaje de conocimientos, creencias y teorías personales a través de las cuales interpretan y otorgan sentido a su práctica cotidiana; - Lo que los profesores piensan y saben es relevante para comprender las decisiones y acciones que toman en la clase; - Los saberes docentes son plurales y heterogéneos, provienen de diversas fuentes y se adquieren en el tiempo en un proceso en el que convergen factores biográficos, institucionales, sociales, culturales, etc. - Los saberes que ponen en juego los docentes presentan ciertas características en común: son inherentes a la profesión, son tácitos, particulares y subjetivos adquiridos a través de la experiencia cotidiana. (p. 58)
\end{abstract}

Cabe constatar que estos saberes o campos del saber también podrían denominarse competencias profesionales (Perrenoud, 2007) o estándares de desempeño y formación (Meckes, 2014). El concepto estándares es utilizado por países de tradición anglófona (Estados Unidos, Inglaterra, Escocia, Australia, Nueva Zelanda) y el de competencias, por países europeos, Canadá y algunos países de Latinoamérica. Siguiendo a Meckes (2014), el estándar debe “incluir tanto el saber como el saber cómo, las disposiciones o valoraciones y el ser capaz de hacer” (p. 58). Algunos países de Latinoamérica han avanzado en la construcción de los estándares básicos en la formación inicial docente para la orientación de los procesos formativos, de acuerdo con el documento presentado por la UNESCO (2014), que aborda temas críticos dirigidos a las políticas de formación docente para América Latina y el Caribe.

¿Es importante la reflexión en la formación docente?, ¿es posible formar en reflexión? Los saberes que van aprendiendo las y los docentes y que serán aplicados a las prácticas de acción del mañana, requieren de reflexión constante. La reflexión es un proceso mental que relaciona el conocimiento y la acción. Puede ser un examen o la construcción de una argumentación de lo que se prepara, hace o deja de hacer. Así, se espera mantener en el tiempo una práctica reflexiva desarrollada por las y los docentes.

Para Freire (1970) abordar la reflexión y, especialmente la reflexión crítica sobre la práctica (praxis) es una parte importante en la formación docente, consiste en la práctica de analizar la práctica para que la acción no sea un mero activismo. Perrenoud (2007), apunta que "en formación profesional, todos los 
conocimientos son en última instancia de orden práctico, si se admite que la práctica es también una reflexión en la acción y sobre la acción" (p. 25). Entonces, es necesario desarrollar la práctica reflexiva en el oficio docente considerando, según Perrenoud (2004), "la autonomía y la responsabilidad de un profesional no se entienden sin una gran capacidad de reflexionar en la acción y sobre la acción” (p. 12). Además, considera que para:

Una verdadera práctica reflexiva, es necesario que esta postura se convierta en algo casi permanente y se inscriba dentro de una relación analítica con la acción que se convierte en algo relativamente independiente de los obstáculos que aparecen o de las decepciones. (Perrenoud, 2004, p. 13)

Schön (1983) argumenta que el/la docente, además de aplicar contenidos, metodologías y didácticas, a lo que denomina conocimiento en la acción, necesita procesos de reflexión. Se refiere principalmente a pasar desde una racionalidad práctica o técnica a otra, donde la reflexión profesional es el camino para mejorar sus propias prácticas. Para Schön (1983), "nuestro conocimiento es de ordinario tácito, está implícito en nuestros patrones de acción y en nuestra sensación respecto a las cosas con las que tratamos. Parece correcto decir que nuestro conocimiento se da desde nuestra acción" (p.55). Así, la acción se complementa con la reflexión y permite un saber orientado a remediar contextos y proponer alternativas de cambio a través de la acción experimentada.

La reflexión, para Perrenound (2004), es necesaria en todas las competencias profesionales y de cada oficio. Debe ser inseparable de la formación inicial y la relación teoría- práctica. Para ello, se requieren actitudes, saber hacer, método y posturas reflexivas.

La formación docente, necesariamente, debe estar orientada a la acción, pero no sola, sino acompañada de una reflexión sobre la acción y para redirigir y mejorar una acción intencionada. Además, la reflexión ineludiblemente debe tener una conexión importante con las teorías que subyacen y orientan dichas prácticas. La acción pedagógica debe ser reflexiva, debe ser una tarea que desarrolle experiencias prácticas situadas basadas en saberes, relación que pueda entregar a la actividad pedagógica una lógica inmanente de acción reflexión.

Aquél que indaga y requiere responder a sus inquietudes permitirá aprender de sus hallazgos y de sus aciertos y errores. Vezub (2016) considera que la reflexión, la indagación y el cuestionamiento, ayudan a mejorar la enseñanza. Otro aporte lo desarrolla Heidegger (2000), considera que:

El pensar no se convierte en acción porque salga de él un efecto o porque pueda ser utilizado. El pensar solo actúa en la medida en que piensa. Este actuar es, seguramente, el más simple, pero también el más elevado, porque atañe a la relación del ser con el hombre. (p.12)

Un practicante reflexivo, señala Perrenoud (2007), plantea preguntas sobre su tarea, las estrategias más adecuadas, los medios que deben reunirse y el programa de tiempo que debe considerarse. La reflexión, para acumular saberes porque permite aprendizajes de sí y del medio; colabora con la preparación para una responsabilidad política y ética; permite la cooperación y aumenta la capacidad de innovación en las prácticas y finalmente:

en cualquier caso, la práctica reflexiva se aprende con un entrenamiento intensivo, lo que nos remite no tanto al pequeño módulo de iniciación a la reflexividad, sino a las formaciones completas orientadas al análisis de prácticas y al procedimiento clínico de formación. (p. 44)

Ahora, desde el punto de vista de Gil (2018), quien considera la necesidad de un paradigma reflexivo crítico necesario para la formación docente, insiste en que:

Es la acción reflexiva compartida que permite a quienes están en formación, construir sus propios estilos y formas y no adoptar otras predeterminadas. Se basa, por ello, en un proceso de acompañamiento del profesorado para ayudarle a encontrar progresivamente sus propios caminos en la enseñanza. La consistencia de esta formación demanda identificar las tareas y 
competencias que el profesorado debe efectuar y los conocimientos integrados que necesita para el ejercicio profesional. (p.290)

Respecto al tema de las relaciones complejas entre reflexión y acción, aparecen algunas áreas denominadas las dimensiones de complejidad a considerar en la formación inicial docente, señala Aravena (2016) que es posible constatar tres dimensiones que se relacionan constantemente y cada una puede estudiarse separada de la otra, pero, ninguna de ellas por sí solas comprende la totalidad y la complejidad del campo de la educación:

a. A nivel teórico, se ubican aquellos actores portadores de los saberes educativos. Por ejemplo, pedagogos e investigadores educativos u otros actores que estén reflexionando y aportando intelectualmente a la configuración del campo educativo. b. A nivel legal. Aquí se ubican las políticas públicas, las reformas y reglamentos destinados a las instituciones educativas. c. Las prácticas escolares, son aquellas que se desarrollan al interior de la escuela. Aquí confluyen los distintos actores (educadores y otros profesionales ligados a la educación) que día a día participan en el proceso de enseñanza aprendizaje. (p. 132)

La población docente es preparada para aplicar su actividad en estos tres niveles, seguramente están más en las prácticas escolares, pero se valen de la teoría, especialmente educativa y de la legalidad (distintos aspectos de las políticas educativas) para desarrollar su práctica educativa. Los tres niveles le dan sentido a la profesión, la configuran a nivel teórico y práctico.

La necesidad de reflexión requiere, estar constantemente interrogando y preguntándose qué, cómo y por qué pasan ciertas cosas y no otras; se pregunta sobre los sujetos educativos (niños y niñas, educadores y educadoras, directivos, madres y padres), también sobre las políticas educativas, las instituciones educativas y cómo se relacionan con su rol docente. Además, se interroga sobre las teorías educativas, las estudia, las conoce, las aplica y las condiciona y resignifica su labor.

\section{2- LOS TEMAS EMERGENTES EN EDUCACIÓN ¿CUÁLES Y QUIÉN LOS INSTALA EN LA FORMACIÓN DOCENTE? INTERROGANTES PARA REFLEXIONAR PERMANENTEMENTE.}

Anteriormente se ha puesto la relevancia de la formación docente, en relación con los saberes, a la reflexión, a la ubicación en relación con las políticas, las prácticas y la legalidad. Desde el punto de vista de la pregunta, existen otros cuestionamientos, discusiones y situaciones que están presentes en las sociedades y que ponen al/la docente en tensión.

¿Quién orienta los temas emergentes? ¿Por qué son emergentes? ¿Temas emergentes de qué?, ¿para quién? Cada uno de los temas emergentes se relaciona con los países, localidades, políticas educativas, familias, ciudades, escuelas, niños y niñas y adolescentes. Existen un sin número de temas emergentes que dependen de variables sociales, económicas, políticas, familiares, contextuales, hoy sanitarias, etc. Por ejemplo: innovación, diversidad, tecnología, cultura digital, de diversidad y género, etc. Seguramente, incluso hay algunos que no se abordan en este texto. Pero, lo relevante es apreciar que estos temas están presentes y que condicionan la labor docente y su relación con estas temáticas.

Podría señalarse también que hay temas emergentes que pueden venir de organismos internacionales, por ejemplo; de la OCDE, Unesco, Banco Mundial, que ponen en el tapete ciertos cuestionamientos y los lleva a los países tanto desarrollados como subdesarrollados a condicionar su actuar. No se está señalando que sean temas menos importantes, sino que son temas que les interesan a las agencias internacionales.

Los temas de interés a la educación, a las escuelas, a niños y niñas, y a la población docente, que provienen de la misma práctica, de la misma relación humana que se da día a día. Y que son posibles de visualizar, de reflexionar y por qué no decirlo, de investigar.

El y la docente, se ubican en un lugar excepcional para preguntar-se, observar, reflexionar, investigar y construir conocimiento con base en las características sociales, culturales, políticas de la escuela. El preguntarse por qué afectan o conmueven la docencia, revisar y conocer con qué temas puede formarse y trabajar y cuáles, simplemente le son lejanos, pero no por ello ausentes. 
Habría que interrogar-se por las y los actores de la educación, por los niños y niñas; por las y los educadores; por la relación y disposición de la población docente a la educación; por las pertinencias de las acciones pedagógicas en relación al entorno socio cultural. Indagar también, en qué es ser niño, adolescente o joven en el siglo XXI; por las minorías étnicas, sexuales, inmigrantes; por la diversidad y las discusiones de género; por las culturas y por la paz.

Los temas, no pretenden agotarse y dependerán de los contextos socioculturales en el que el/la docente desarrolle su ejercicio profesional. La idea es sólo presentar y describir brevemente algunas temáticas emergentes, no se pretende instalar como las relevantes de hoy, sino más bien, destacar la existencia de diversidades temáticas que hacen la labor del/la docente contemporáneo compleja, desafiante en permanente reinvención a partir del dinamismo social existente.

\section{a. Tecnología}

La cultura digital, el ciberespacio, la mass media (medios de comunicación de masa como cine, radio o prensa), representan la era de las tecnologías (Castells 2014, 2020; Polo, 2020). La tecnología es el conjunto de elementos que colaboran a la vida diaria y a solucionar problemas o son un medio para la facilitación de experiencias educativas. ¿Qué relación tiene el/la docente con la tecnología?, ¿cómo la utiliza?, ¿se prepara para la cultura digital?, ¿comprende la relación entre docencia, cultura digital y niños - niñas digitalizados?

La tecnología provee acceso a la comunicación y comunicación en tiempo y espacio. ¿Requiere de educadores formados? La tecnología ayuda a potenciar distintos procesos como: el aprendizaje y pueden hacerlo más atractivo; estimulan habilidades y destrezas para buscar las mejores decisiones que permitan preferentemente solucionar problemas reales y prácticos. Sin embargo, ponen el desafío sobre: ¿qué camino se utiliza?, ¿cómo se desarrollan habilidades para que la tecnología sea un medio que permita llegar al conocimiento?

¿Cómo razonan niños y niñas digitalmente?, son los niños y niñas similares a los de la década pasada, respecto de la familia, posiblemente son hijos de padres millennials ${ }^{[1]}$ o, que se enmarcan en la generación $\mathrm{Z}^{[2]}$ o son de la generación Alpha ${ }^{[3]}$. Niños y niñas que probablemente nacieron cerca de un celular, una tablet, un computador, internet, comunicación virtual.

¿Qué relación tienen niños y niñas con las redes sociales, cómo las usan, la virtualidad es parte de la realidad? ¿Cómo utilizan los juegos en red? Al ser juegos diacrónicos y sincrónicos, ¿qué aprenden y cómo aprenden a desarrollarlo? ¿Qué papel juegan los adultos en esa relación de la infancia con la tecnología? ¿La familia, qué dice al respecto? ¿Se involucra? ¿Estar juntos en una casa, es estar juntos realmente?, ¿qué implicancia tiene la realidad virtual para un niño (a) de 4 año? Las redes sociales, la cultura del hedonismo, las fotos, cómo se entiende la amistad, la familia; qué se entiende por el lenguaje, cuál lenguaje, los argos, emoticones, cómo se comunican las personas, qué pasa con la gramática, el lenguaje verbal y no verbal, ¿qué le pasa a la escuela con todo esto?, es parte de la primera infancia, ¿qué rol tienen las y los docentes con la tecnología? Son muchas las interrogantes a realizar y hacerse por parte del educador, Sin duda, condiciona su ser docente.

\section{B. La Migración}

El desplazamiento de las personas de un lugar a otro, es un tema recurrente en los países latinoamericanos (Rodríguez, 2017). Se reconoce una la alta migración hacia el norte y hacia el sur de América. Según el Ministerio de Relaciones Exteriores de Colombia (2020), las cifras señalan que más de un millón de migrantes venezolanos estarían en territorio colombiano. Esta situación ha provocado un cambio en la región Andina. La migración, si bien es un fenómeno que ha tenido una tendencia masiva, se ha registrado durante la historia de la humanidad. 
Los desplazamientos se han realizado desde el centro al norte, principalmente a Estados Unidos y al sur de América, a países como Chile, Argentina, Brasil y Perú. Son personas que buscan mejorar sus condiciones de vida y se insertan con o sin familia en un contexto sociocultural diferente. Sin duda, la migración condiciona el quehacer de la educación.

\section{Los Desplazados}

Para Colombia este es un problema con características históricas por las décadas en que se mantiene este conflicto interno. Un dato de gran magnitud es que sitúa a este país como aquel que tiene el mayor desplazamiento interno. Más de 7 millones de personas se encuentran bajo esta situación (Alto Comisionado de las Naciones Unidas para los Refugiados [ACNUR], (2019). Esto, provocado por el conflicto armado, donde grupos de guerrilleros obligan a las familias a dejar sus tierras. Una salida forzada donde la gente, principalmente indígenas, negros, campesinos y pobres, deben emigrar hacia otros destinos dejando atrás no solo sus pertenencias, sino su vida, sus costumbres y su cultura.

El desplazamiento desarrolla un efecto profundo en la familia, en niños y niñas y en la sociedad. ¿Cómo responde ante esta situación el sector educativo?, ante nuevas demandas socioeducativas ¿cómo dar cabida a todos los niños y niñas con dificultades por desarraigo, bajo nivel educativo, extra edad, traumas, etc.? Claramente, son necesarias las respuestas, tanto los gobierno como los Ministerios de Educación deben ser garantes de que los procesos educativos se desarrollen en todos los niños y niñas, sin excepción.

\section{Pueblos Indigenas}

Latinoamérica se caracteriza por la presencia de grupos étnicos, la mayoría preincaicos. A lo largo del continente se puede dar cuenta de ellos. ¿Cómo se reconocen? Según estadísticas, se puede reconocer, a lo menos, 522 pueblos indígenas, desde la Patagonia Chilena y Argentina hasta el norte de México, de mar a cordillera, desde el Pacífico al Atlántico, pasando por la Amazonía, los Andes y el Caribe. Cabe preguntarse entonces, si la población docente requiere de conocimientos y saberes interculturales, que según Walsh (2007), le permitan reconocer las relaciones interétnicas, las formas críticas de pensar en la historia y reafirmadas por la colonia.

La América Latina de hoy la caracteriza la presencia de los pueblos indígenas. Algunos de ellos, están presentes en varios países como los Aymaras y los Mapuches. ${ }^{[4] ~ ¿ D o ́ n d e ~ e s t a ́ n ? ~ L a s ~ c u l t u r a s ~ a n c e s t r a l e s ~}$ están en todos los territorios de América Latina. Producto de la colonización algunos viven en comunidades manteniendo su cultura y muchas personas emigraron a las ciudades. Cada país tiene una política respecto a las comunidades y sus territorios. ¿Cómo se trabaja con las culturas indígenas, con su lengua, su cosmovisión de mundo?, ¿qué idioma se usa?, ¿cómo se trabaja con su territorio y costumbres? ¿La educación formal, recoge los aspectos culturales de idioma? Walsh (2013), desarrolla la comprensión pedagógica sobre la relación, las culturas, la identidad, la memoria con un intento intercultural y decolonial.

\section{E. La Etnoeducación}

Reconociendo la diversidad de pueblos y culturas latinoamericanas, la etnoeducación pone su mirada en grupos y comunidades indígenas, haciendo que los propios sujetos tengan voz y se transformen en partícipes de su propia educación. Así, se valoran los conocimientos, características e intereses de los pueblos, manteniendo la identidad y la cultura. Para Artunduaga (1997), "la etnoeducación es un proceso 
de recuperación, valoración, generación y apropiación de medios de vida que responde a las necesidades y características que le plantea al hombre su condición de persona" (p.1).

La etnoeducación requiere de respuestas pedagógicas coherentes para el enriquecimiento de la sociedad. Vale la pena preguntarse entonces ¿cómo se desarrolla en las instituciones?, ¿se respetan las culturas?, ¿cómo se trabaja con las distintas culturas?, ¿cómo desarrolla el respeto, el diálogo, la tolerancia?, ¿es la etnoeducación el camino posible para la integración cultural?

\section{F. Afrodescendientes}

Son aquellas personas de origen africano o que sus ascendientes lo fueron. Principalmente llegaron a América producto de la esclavitud transatlántica y el tráfico de personas, especialmente en los siglos XVI y XIX. Según la Comisión Económica para América Latina y el Caribe [CEPAL] (2017), Brasil sería el país con más afrodescendientes (50,9\%), seguidos por Cuba (35,9\%), Puerto Rico (12,4\%), Estados Unidos (13\%), Colombia (10,5\%), Panamá (8,88\%), Uruguay (4,6\%) Costa Rica (7.8\%) y Ecuador (7,2\%) (p. 52 y 55). Por otro, cabe constatar que según el Banco Mundial (2018), la población se concentra principalmente en las ciudades (80\%).

Hoy, la población afrodescendiente habita los distintos países de Centroamérica y Suramérica. Habrá que preguntarse entonces por la educación. ¿Reconoce las características de la población Afro?, ¿qué pasa en las aulas con el respecto de las identidades de niños y niñas?, ¿es la educación una posibilidad de integración?, ¿cómo se puede enriquecer las culturas locales a partir de las tradiciones que estas personas tienen?

\section{G. Educación para la paz}

¿Se educa para la paz?, ¿quién o quiénes deberían hacerlo?, ¿es la educación para la paz una posibilidad para transformar la sociedad? Sin duda, la paz como valor universal es deseable para todos. Mantenerla es un fin para todas las sociedades. Es entonces posible pensar en una pedagogía de y para la paz. Dejar atrás episodios de violencia, dolor y odio de guerra y guerrilla que destruyen la sociedad y generan temor y pánico en las personas. Construir posibilidades de paz y armonía es posible, así como también educar para la paz. Así lo considera Manjarrés y Molano (2001) "la construcción de la paz es una obra permanente, multidimensional y dinámica, que requiere el enraizamiento de valores pacíficos en la población” (p. 34).

Los gobiernos son responsables de las distintas políticas educativas y sociales dirigidas hacia la paz social. ¿Está la población docente formado para la paz?, ¿cuál es su rol en la sociedad?, ¿está el y la docente llamados a educar para la paz o, es un tema de otros? Y entonces, ¿cómo se hace?, ¿es necesaria y alcanzable la paz en todos los territorios?

\section{H. La Sustentabilidad}

En pleno siglo XXI es un tema en cuestión. Se han realizado cumbres sobre el desarrollo sostenible, agencias nacionales e internacionales dan cuenta de la problemática de la tierra y del cambio climático. ¿Qué entienden las agencias internacionales, el Estado, los empresarios, la familia, el niño y la niña? ¿ De quién es la responsabilidad? Por una parte, de cada ciudadana y ciudadano, pero la más importante, es de los Estados los que no ponen cuidado, permitiendo que se sobre explote sin conciencia y los empresarios, sacan los recursos naturales de los países con poco criterio. ¿Cómo se forma la conciencia ambiental?, ¿cómo desarrollar una educación que vaya más allá de la conciencia ambiental y se transforme en una experiencia sostenible en el tiempo? 


\section{Diversidad, género, sexismo y no discriminación}

Son temas que hoy cobran una importante reflexión en la sociedad. Las actitudes, conductas, valores, creencias socialmente formadas y que dependen de la familia y de los contextos socioculturales. Son cuestiones que también están en la escuela y que deben ser consideradas en la tarea docente.

La igualdad de género y los derechos humanos presentan argumentos éticos fundamentales como la libertad, igualdad, respeto, solidaridad y diálogo, como forma de resolución de conflictos (Cortina, 1997). Promover la convivencia sana, respetuosa de las diferencias de género, de sexos, hacia la no discriminación. ¿Está la población docente preparado en estos saberes? ¿Se aborda en la formación docente una cultura de los derechos humanos? Ciertamente peguntar-se generaría respuestas reflexivas que orientan sus prácticas hacia una sociedad diversa y que valore las diferencias.

\section{J. Rol/Función /Tarea/ Labor/ Práctica docente}

Preguntar-se constantemente por el rol docente implica tener claridad sobre la esencia de éste. Implica reconocer -se en aquella acción - reflexión que le da sentido a la acción. Giroux (1997), examina el conocimiento disciplinar, las capacidades y subjetividades, así como los principales contenidos educativos y curriculares:

Los docentes deben tener un control teórico de las formas en que se construye la diferencia, ya que ésta puede adoptar diversas representaciones y prácticas que nombran, legitiman, marginan y excluyen las voces de los grupos subordinados. Este control teórico permitirá al docente trabajar los contenidos educativos de manera pertinente para el logro de los fines educativos ya esbozados, pero el logro efectivo de tales fines exige que el docente se constituya en aquel que cruza fronteras para legitimar la diferencia como una condición básica para entender los límites del propio conocimiento. (González, 2006. pp. 84-85).

Así, Giroux (1997) señala que "los profesores necesitan desarrollar un discurso y un conjunto de hipótesis que les permitan actuar más específicamente como intelectuales transformativos” (p. 36), de esta manera potenciar la acción y la reflexión.

Ser docente abre posibilidades de preguntarse desde dónde parto y hasta dónde quiero llegar. De esta manera, trazar una posible ruta de tránsito orientado con las estrategias que poseo para desarrollar los objetivos con los y las estudiantes. ¿Entonces quién o quiénes configuran el rol docente? ¿La institución educativa?, ¿las políticas educativas?, ¿la acción educativa? Entonces, preguntar-se por el rol/función/tarea/ labor o práctica docente en este siglo, sería clave para comprender la educación, la escuela y la sociedad contemporánea.

\section{REFLEXiOnes FinAles}

Preguntar-se en la formación docente lleva a poner a la pregunta en un lugar privilegiado en la formación. La duda, la interrogación debiera ser el lugar de toda formación en tiempos, en los cuales las certezas en la sociedad y en la educación en particular, se han ido diluyendo en detrimento de la institución escolar, que representaba hace menos de un siglo el templo de un saber sabio, hoy se considera que ya no lo es.

El artesano moldea su obra tal como lo hace el educador. La obra de educadoras y educadores es inconmensurable e intangible. Es necesario que se valorare en el tiempo y en el espacio el aporte que realizan a la sociedad, especialmente en tiempos en los cuales la creación e innovación pueden y deben instalar nuevos desafíos y hacerse cargo de nuevas temáticas emergentes. La importancia de trabajar con preguntas y temas emergentes, permite reflexionar y aprender de la experiencia. Permite a los y las docentes crecer en la formación de su oficio y le permite adaptarse de mejor manera a los vertiginosos cambios. 
La cotidianeidad pone temas relevantes, urgentes, posibles para poder trabajarlos. Preguntar-se cómo, qué, por qué, permite pasar de una racionalidad instrumental-técnica a una sustantiva, que trabaje por el ser humano. Reconocer el espacio, el trabajo personal y social, requiere de conocimiento y aprendizajes.

Habermas (2015), invita a la apertura del mundo de la vida, a través del lenguaje entre los distintos actores es posible reconstruir el sentido de la vida. El autor separa el mundo de la vida al ámbito material, donde estaría el dominio de las operaciones instrumentales y de la naturaleza. En el ámbito simbólico, es el lugar en que los sujetos se comunican, tejen sus vidas, negocian, interpretan el mundo a través del uso del lenguaje. En la era moderna, mundo de la vida y sistema se contraponen. En el mundo de la vida está la familia, la cultura y sociedad. En el sistema están todas las instituciones, incluida la escuela. ¿Qué hacer, para que esos dos mundos convivan?, ¿es posible llevar el mundo de la vida al sistema? Por cierto, es un gran desafío.

Finalmente, la formulación de preguntas en la formación docente favorece en construir y orientar realidades y contextos. Ayuda a formular supuestos y a avanzar en el conocimiento. Preguntar-se, acerca el conocimiento a las y los docentes, ayuda a la reformulación de sus ideas y a su ejercicio profesional reflexivo, para así poder resignificar la práctica docente. Interrogar y preguntar-se permite re-conocer la obra de educar, de levantar inquietudes y poder responderlas en la práctica, trabajando de manera permanente e inmanente con las teorías educativas y sociales. Este desafío en la formación recién comienza, y muy probablemente, la única certeza es que la tarea - rol docente- tendrá constantes cambios en una sociedad en que todo lo sólido se desvanece en el aire.

\section{Referencias bibliográficas}

Alto Comisionado de las Naciones Unidas para los Refugiados [ACNUR]. (2019).Migración, desplazamiento y educación. Construyendo puentes, no muros. Francia: UNESCO.

Aravena, M. (2016).Formación Inicial Docente en Investigación Educativa: Estudio de Caso de la Formación en Investigación en las Escuelas de Pedagogía Básica Chilenas. (Tesis de doctorado). Universidad Nacional de la Plata, Argentina. Recuperado de https://bit.ly/2VoVnT5

Artunduaga, L. (1997).La etnoeducación: una dimensión de trabajo para la educación en comunidades indígenas de Colombia. Revista Iberoamericana de educación, (13). Recuperado de https://rieoei.org/historico/oeivirt/rie13 a02.htm

Banco Mundial. (2018). Afrodescendientes en América Latina: hacia un marco de inclusión. Estados Unidos: Banco Mundial. Recuperado de https://n9.cl/8ouw

Castells, M. (2014). El impacto de internet en la sociedad: una perspectiva global. En M. Castells (Ed.), C@mbio: 19 ensayos clave acerca de cómo Internet está cambiando nuestras vidas, (pp. 9-24). Madrid: BBVA-OpenMind. Recuperado de https://n9.cl/8a8b

Castells, M. (2020). La nueva sociedad red. La Vanguardia. Recuperado de https://n9.cl/85mb5

Comisión Económica para América Latina y el Caribe [CEPAL]. (2017). Situación de las personas afrodescendientes en América Latina y desafíos de politicas para la garantía de sus derechos. Chile: Naciones Unidas. Recuperado de https://n9.cl/umng

Cortina, A. (1997). Resolver conflictos, hacer justicia. Cuadernos de pedagogia, (257), 54-56.

Fanfani, T. (2006).El oficio de docente. Vocación, trabajo y profesión en el Siglo XXI. Argentina: Siglo XXI

Freire, P. (1970). Pedagogía del oprimido. España: Siglo XXI Ediciones S.A.

Gil, Rafael. (2018).La formación docente: horizontes y rutas de innovación. Argentina: CLACSO.

Giroux, H. (1997).Los profesores como intelectuales. Hacia una pedagogía crítica del aprendizaje. España: Paidós.

González, L. (2006) La Pedagogía Crítica de Henry A. Giroux. Revista Electrónica Sinéctica, (29), 83-87.

Habermas, J. (2015).Mundo de la vida, política y religión. España: Trotta.

Heidegger, M. (2000).Carta sobre el humanismo. España: Alianza. 
Marcela Angelina Aravena Domich. Preguntar-Se en el oficio de educar: la importancia de la Pregunt...

Larrosa, J. (2020).El profesor Artesano. Materiales para conversar sobre el oficio. España: Laertes

Manjarrés, M. y Molano, M. (2001).La escuela que los niñosperciben: aportespara construir una cultura desde una gestión institucional. Colombia: Ed. Universidad Pontificia Bolivariana.

Ministerio de Relaciones Exteriores de Colombia. (2020). Más de un Millón 825 mil venezolanos estarían radicados en Colombia. Recuperado de www.migracioncolombia.gov.co

Meckes, L. (2014). Estándares y formación docente inicial. En Oreal/Unesco (Eds.), Temas criticos para formular nuevas politicas docentes en América Latina y el Caribe: el debate actual. (pp. 53-110). Chile: Orealc/Unesco. Recuperado de https://bit.ly/32SGTA5

Perrenoud, P. (2004).Diez nuevas competencias para enseñar. España: Graó. Recuperado de https://www.uv.mx/dgda ie/files/2013/09/Philippe-Perrenoud-Diez-nuevas-competencias-para-ensenar.pdf

Perrenoud, P. (2007).Desarrollar la práctica reflexiva en el oficio de enseñar: profesionalización y razón pedagógica. México: Graó

Polo, A (2020).Sociedad de la Información, Sociedad Digital, Sociedad de Control. Revista Vasca de sociología y ciencia politica, (68), 50 - 77.

Porta, M. (2019) Los saberes docentes: enfoques y perspectivas teóricas. (tesis de posgrado). Universidad Nacional de San Luis, Argentina. Recuperado de http://fchportaldigital.unsl.edu.ar/index.php/TESIS/article/view/221/145

Rodríguez, J. (2017).Efectos de la migración interna sobre el sistema de asentamientos humanos de América Latina y el Caribe. Revista de la CEPAL, (123), 7-34.

Sánchez, T. y González, H. (2016). Saber pedagógico: fundamento del ejercicio docente. Revista Universidad de la Sabana, 79(2), 241-253.doi:https://dx.doi.org/10.5294/edu.2016.19.24

Schön, D. (1983).El profesional reflexivo. Cómo piensan los profesionales cuando actúan. España: Paidós.

Sennett, R. (2009).El Artesano. España: Anagrama.

Tardif, M. (2004).Los saberes del docente y su desarrollo profesional. España: Narcea.

Tonucci, F. (2 de mayo de 2018).La misión principal de la escuela ya no es enseñar cosas.WEB del maestro CMF. Recuperado de https://n9.cl/1h78

Toledo, V (2020). Generación Z. El mundo tal y como lo conocemos. Trabajo de fin de máster. España: Universidad de Pamplona. Recuperado de https://n9.cl/6wg7s

Vezub, L. (2016). Los saberes docentes en la formación inicial. La perspectiva de los formadores. Pensamiento educativo. Revista de Investigación Educacional Latinoamericana, 53(1),1-14.doi http://dx.doi.org/10.7764/P EL.53.1.2016.9

Walsh, C. (2007). Interculturalidad, colonialidad y educación. Revista educación y pedagogía, 19(48) ,25-35. Recuperado de https://n9.cl/r8hf

Walsh, C. (Ed.) (2013).Pedagogias decoloniales. Prácticas insurgente de resistir, (re) existir y (re) vivir. Tomo I. Recuperado de https://n9.cl/axtj

\section{Notas}

[1] La generación Millennials es la generación del milenio, nacidos entre 1981 y 1999. Sigue a la generación X y precede a la generación $\mathrm{Z}$.

[2] La generación Z, es post Millennials, nacidos a finales de la década de los 90’ y principios del 2000.

[3] La Generación Alpha caracteriza los nativos digitales. Después de la Generación Z formada por aquellos nacidos entre 1994 y 2010. Los expertos comienzan a hablar de una nueva generación en plena era digital a la quehan bautizado como la Alpha, formada por los nacidos a partir de 2010 los hijos de la Generación X y los millennials (Toledo 2020).

[4] En el caso de Chile, los principales serían los Mapuches, Atacameños, Colla, Quechua, Rapanui, Yagan, entre otros. Argentina: se encuentran las culturas Tobas, Onas, Yaganes, Araucanos, Tehuelches, entre otras. En Perú: Aymaras, Awajun, Amahuaca, Amrakaeri, entre otros. Bolivia: Aymara y Quechua hablante, Guaraníes y Mojeños. En Uruguay: Churrúas, En Paraguay: Guaraníes. Brasil tendría unas 305 tribus. Guaraníes, Yomamanis, Tikunas y los kaingangs, o en el árido interior del nordeste, como los Pataxohãhãhães y los Tupinambás. En Ecuador: Quichuas, Montubio, Shuar, 
Revista Educación, 2021, vol. 45, NúM. 1, Enero-Junio, ISSN: 0379-7082 2215-2644

Salasacas, entre otras. Venezuela se puede encontrar varias culturas como: Arawako, Bari, Akaway entre otras. México: Nahuas, Mayas, Zapotecas, Mixtecas, Otomíes, Totonacas, entre otros. 